\title{
Régimen directo y preposicional del verbo cuidar en castellano: una aproximación histórica*
}

\author{
Francisca Toro Varela*
}

\begin{abstract}
Resumen

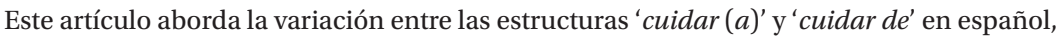
entendidas como régimen directo y preposicional respectivamente, desde una perspectiva diacrónica. El objetivo del estudio es demostrar que un cambio diacrónico que cruza la caracterización semántica y sintáctica de los verbos cuidar y curar permite explicar la actual alternancia de cuidar entre ambas estructuras. Para ello se revisó en el Corpus Diacrónico del Español (CORDE) las estructuras de régimen directo y preposicional de los verbos cuidar y curar, comprendidas entre los siglos XII y XV. Se concluye que la transferencia semántica de curar a cuidar ocurrida durante finales de la Edad Media induce, al mismo tiempo, a un cambio sintáctico, en tanto cuidar empieza a admitir objetos animados en construcciones de régimen directo, lo cual en el período más temprano estaba limitado a curar.
\end{abstract}

Palabras clave: régimen directo, régimen preposicional, transitividad, cambio diacrónico.

\section{Direct and prepositional regimen of the verb cuidar in spanish: an historical approach}

\begin{abstract}
This article addresses the variation between the Spanish structures 'cuidar (a)' and 'cuidar $d e$, understood as direct and prepositional regimen respectively, from a diachronic perspective. The aim of this study is to demonstrate that a diachronic change that crosses the semantic and syntactic characterization of the verbs cuidar and curar allows explaining the current alternation of cuidar between both structures. The Methodology consisted of the revision of Corpus Diacrónico del Español (CORDE), in order to analyse the direct regimen and prepositional structures of the verbs cuidar and curar, between the $12^{\text {th }}$ and $15^{\text {th }}$ centuries. It was concluded that the semantic transfer from curar to cuidar occurring during the late Middle Ages induces, at the same time, a syntactic change. Namely, it can be said that cuidar begins to admit animated objects in direct regimen constructions, which in the earlier period was only restricted to curar.
\end{abstract}

Keywords: direct regimen, prepositional regimen, transitivity, diachronic change.

* Chilena. Licenciada en Letras mención Lingüística y Literatura Hispánica de la Pontificia Universidad Católica de Chile. Estudiante de Magíster en Letras mención Lingüística, PUC. Santiago, Chile. fptoro@ uc.cl 


\section{Introducción}

En su variante transitiva, el verbo cuidar en español acepta, al menos, dos clases de construcciones, bien un complemento directo tradicional (en adelante, CD), como en (1a), o bien un complemento preposicional (en adelante, CPREP) encabezado por $d e$, como en (1b):

(1a) Cuidar a una persona.

(1b) Cuidar de una persona.

Ambas construcciones, por lo demás, parecen estar extendidas en todo el mundo hispano, tal como muestran los siguientes ejemplos:

(2a) También tendremos que cuidar nuestras palabras (Venezuela, CREA).

(2b) Se dedicaba últimamente a cuidar una docena de gatos (España, CREA).

(2c) Somos los llamados a cuidar de lo nuestro (Colombia, CREA).

(2d) Pero lo más bonito era que le gustaba cuidar de uno (Chile, CREA).

Si bien una de ellas es más frecuente que la otra (47 casos con la primera frente a 5 con la segunda, según la base de datos ADESSE), cabe preguntarse cómo se relaciona este par y si existe una motivación histórica para la alternancia. Tal inquietud surge especialmente si se considera su etimología: mientras que cuidar habría heredado su doble construcción del verbo latino cōgitāre (Corominas, 1987) a partir del cual se desarrolla fonéticamente, en esta evolución opera un cambio semántico, pues cōgitāre significaba 'pensar' (Glare, 1968, p.344) en el período clásico; en cambio, su significado parece estar relacionado con otro verbo, cūrāre, del cual deriva el español curar y cuyo funcionamiento sintáctico presenta algunas diferencias, como se verá a continuación, que vale la pena considerar para comprender el desarrollo de cuidar, ya que a menudo los cambios semánticos no ocurren de manera aislada (Cano, 1977).

Así, el objetivo general de esta investigación es determinar, en términos generales, si la alternancia entre CPREP y CD del verbo cuidar puede explicarse de algún otro modo que no sea la pervivencia de la alternancia en cōgitāre; y en términos específicos, si tal alternancia se puede justificar a partir de la influencia del desarrollo paralelo del verbo cūrāre. Para esto, los pasos seguidos durante la investigación han sido: establecer los rasgos semánticos del segundo argumento de los verbos cuidar y curar, y 
caracterizar el comportamiento semántico-sintáctico de los verbos cuidar y curar de acuerdo a las propiedades del segundo argumento.

\section{Fundamentos Teóricos}

\subsection{Transitividad, rección y rasgos semánticos de la red argumental}

Ciertos acercamientos tradicionales consideran como intransitivos los usos del verbo cuidar con complemento preposicional (así, por ejemplo, Real Academia Española, 2014, 23 ${ }^{\text {a }}$ ed.), lo cual se relaciona con la concepción de transitividad como la propiedad sintáctica que presentan algunos verbos "which require a direct object" (Luraghi \& Parodi, 2008, p.189) y a la propiedad semántica de "transmisión de energía del Sujeto al Objeto" (Torrego, 2010, p. 200). Sin embargo, esta definición no resulta del todo funcional (Torrego), dada la complejidad no solo del concepto de transitividad, sino también de otros asociados a él como el de sujeto y objeto.

Por el contrario, la mejor forma de comprender la variación sintáctica del verbo en cuestión (y las correspondientes consecuencias semánticas) parece ser considerar ambas estructuras como diversas manifestaciones de una cláusula transitiva, en tanto en ambos casos hay un segundo argumento requerido obligatoriamente. En efecto, como señala Candalija (2013, p.183), es posible hablar de transitividad directa y preposicional, dado que es el "tipo de construcción completa [...] el que selecciona tanto el significado como la presencia de la preposición”. Esta aproximación teórica reconoce y afina lo que Cano (1985, p.81) definió como 'transitividad preposicional': "función semántica idéntica entre complementos directos y preposicionales".

En línea con esta concepción, consideramos aquí la transitividad no como una categoría binaria, sino como un continuo escalar a lo largo del cual es posible encontrar infinitas posibilidades (Naess, 2007). Con el fin de cumplir con un criterio de validez tipológica, se entenderá aquí la transitividad no como una característica absoluta del verbo (transitivo o intransitivo), sino como una "scalar notion", donde "sentences and predicates can be more or less transitive” (Luraghi y Parodi, 2008, p.190), en tanto esta definición permite también trabajar con categorías extrapolables a otras lenguas. 
Por otro lado, siguiendo trabajos ya clásicos como el de Hopper y Thompson (1980), no se considerará aquí la transitividad como un rasgo intrínseco del verbo, sino de la cláusula entera, donde la actividad verbal se transfiere desde un 'agente' a un 'paciente', entendiendo estos dos como macrorroles, en línea con propuestas como la de Dowty (1991). De ahí que Alarcos sostenga que más que verbos transitivos o intransitivos, lo que se tiene son predicados transitivos o intransitivos (1966). Este acercamiento permite comprender mejor el modo en que un determinado comportamiento sintáctico del verbo se relaciona con la elección de ciertos rasgos semánticos en su red argumental.

Por su parte, para diferenciar entre las posibles estructuras exigidas por el verbo, hablaremos de rección (cf. Cano, 1985; Martínez, 1987). Así, por ejemplo, el verbo cuidar admite diferentes tipos de rección, entre los cuales se cuentan el sintagma nominal y el sintagma preposicional como segundos argumentos posibles. En este sentido, rección se entiende como las diferentes manifestaciones posibles de la cantidad de argumentos exigidos obligatoriamente por el verbo en un determinado tipo de cláusula.

\subsection{Cōgitāre y cūrāre}

El verbo cuidar se desarrolla fonéticamente a partir del verbo latino cōgitāre 'pensar' (etimológicamente 'perseguir algo en la mente'), un verbo de 'actividad intelectual', lo cual se puede ver en la pervivencia de ciertos valores del verbo cuidar del español moderno (Rivas, 200o). Respecto del cambio semántico diacrónico experimentado por este verbo, Corominas (1987) indica que a partir del siglo XVI cuidar se interpreta con el sentido actual que tenemos en castellano, es decir, 'atender (a alguien o algo $)^{\prime}{ }^{1}$, mientras que en español medieval conserva tal clasificación como verbo típico de actividad intelectual: "S. XVI en el sentido moderno; h. 1140 en su acepción medieval 'pensar"' (p.184). Y es, de hecho, con este significado de 'pensar' que está documentado desde el Poema de Mio Cid (3a) hasta entrado el Siglo de Oro español (3b) (García, 2006).

(3a) así viene esforçado el conde que a manos se le cuidó tomar. (Cantar de Mio Cid, 1140, CORDE)

(3b) yo, cata, creo muy mal, cuido que estavas dormido (Juan del Encina, 1481-1496, CORDE)

1 Nótese que esta acepción ya no permite clasificar al verbo como una actividad de tipo intelectual. 
En cuanto a su comportamiento sintáctico, cōgitāre, al desarrollarse como cuidar, habría mantenido ambas recciones: transitiva y preposicional, en tanto la forma latina también las permitía ${ }^{2}$.

Por su parte, curar se desarrolla fonéticamente a partir de cūrāre 'cuidar, preocuparse por, sanar' (Glare, 1968, p.475) y de él hereda parte de su significado (4a) y también su estructura transitiva, en tanto el verbo español admite un complemento directo típicamente nominal y el verbo latino admitía un acusativo; en cambio, mientras que el verbo latino podía admitir un segundo argumento preposicional ${ }^{3}$ (4b), esta posibilidad desaparece a lo largo de la historia del español.

(4a) Sabet que el açor natural desde ' 1 mes d'agosto ffasta ' 1 mes de novienbre es de curiar, que non sse[a] muy magro ni muy gruesso (Anónimo, c 1300, CORDE)

(4b) vides quanto haec diligentius curem quam aut de rumore aut de Pollione, de Pansa etiam. ('ves con cuánto más interés me preocupo de estas cosas que de los rumores, o de Polión, o de Pansa incluso'. Cic, Epistulae ad Atticum 13.21.3.14).

Como es evidente, estos verbos latinos también admitían más de una rección en su variante transitiva, ante lo cual cabe preguntarse si ello implica diferencias semánticas entre tales construcciones. Si bien no hay registros en la bibliografía del latín sobre estas estructuras particulares, con CD y con de, Baños (2009) sugiere que, al menos, las construcciones de cūrāre con acusativo y dativo (caso de complemento indirecto, CI) experimentan el mismo grado de afectación, dado que se trataría de una diferencia original entre ambas construcciones que resulta neutralizada en la historia de la lengua latina: "la desaparición del Dativo a partir de Plauto sugiere que dicha alternancia no se siente ya semánticamente motivada” (p.135).

\subsection{Rección y rasgos semánticos de la red argumental}

Si bien la estructura con dativo del latín no está relacionada unidireccionalmente con el CPREP en español, este mismo patrón de alternancia

2 Cōgitāre admite variadas construcciones, al igual que muchos otros verbos en latín: con acusativo (aliquid), con ablativo (aliquo), con de + ablativo (de aliquā re), con cláusula relativa, absoluto, infinitivo y $u t+$ subjuntivo (Cf. Perseus Project [en línea]).

3 La construcción con de no era la más común en la época clásica como sí lo era el empleo del acusativo o dativo; sin embargo, el hecho de que la encontremos en Cicerón es prueba de que era un uso coloquial. Probablemente, esta construcción curare de y su perífrasis habere curam de deben de haberse influido recíprocamente. 
sintáctica se habría replicado en la opcionalidad entre régimen directo y preposicional de cuidar en castellano. Para algunos autores, ambas construcciones se comportan como "variantes libres" (Cano, 1977, p. 340) con el mismo grado de afectación. Sin embargo, en línea con trabajos como el de Rivas (2000), cabe preguntarse si los diferentes tipos de rección se relacionan con una variación en el tipo de rasgos semánticos del segundo argumento. Esta autora analiza un conjunto de verbos del español medieval y concluye que "la alternancia de regímenes hace variar sensiblemente el sentido de la construcción, o la definición semántica del segundo actante, o la acepción del verbo" (p. 125). En ese sentido, atribuye el rasgo de /+Animado/ a estructuras con CD, ya que presentan "un objeto 'afectado' por el proceso" (p. 126), mientras que las estructuras con CPREP se identifican con el rasgo /-Animado/, dado que aquí la nota de 'afección' estaría ausente (Rivas, 2000). De manera similar, García-Miguel (1995) apunta que la construcción transitiva es una estrategia explícita de la lengua de expresar un referente afectado por el proceso, "mientras que la construcción preposicional no indica nada explícitamente" (p. 126).

Ahora bien, la noción de afectación como eje de análisis es compleja, puesto que resulta difícil de caracterizar y parametrizar. De allí que para esta investigación hayamos preferido limitarnos a rasgos más concretos como el de animacidad que el de afección, con el fin de detectar alguna posible variación.

\section{Metodología}

La muestra para realizar este estudio fue tomada del Corpus Diacrónico del Español (CORDE) y el rango histórico seleccionado está conformado por los siglos XII, XIII, XIV y XV, correspondientes al período sociocultural de la baja Edad Media. La elección de este período se basa en que se trata del período más antiguo del que contamos con textos cuya lengua podemos afirmar que es ya español o castellano y porque es un tiempo suficiente para visibilizar cambios semánticos y gramaticales. Los límites los constituyen, por un lado, el siglo XII, pues ya podemos hablar de 'castellano antiguo' (y no 'romance') y, por otro, el siglo XV, pues introduce ya rasgos que serán propios del Renacimiento y del español del Siglo de Oro. Cabe destacar que se observaron igualmente los siglos XVI y XVII (pese a que no son reportados aquí) con el fin de evidenciar que los re- 
sultados encontrados tras este estudio se hayan asentado efectivamente en la lengua en los siglos posteriores.

Se rescató de los cuatro siglos observados el número total de casos arrojados con cuidar, por un lado, y curar, por otro. Luego, se calculó la potencia estadística (statistical power) en el programa R-Studio para determinar el tamaño muestral. Según esta prueba, para obtener una potencia alta (normalizada, por convención, en o.8) y un tamaño de efecto ‘bajo' para Ji-cuadrado (que sería el peor de los casos), requeriría una muestra de 1090 casos $^{4}$.

Sin embargo, dado que el número total de los casos de cuidar y curar con CD y CPREP está muy por debajo de esa cantidad: (cuidar tiene en total 103 casos y curar 382 [véanse Tablas 1 y 2 más abajo]), se decidió trabajar con la totalidad de casos del corpus. Así, la muestra finalmente quedó conformada por todos los textos -sin discriminación de géneroque, del siglo XII al XV, presentan los verbos cuidar y curar con las estructuras de régimen directo (cuidar $+\mathrm{CD}$ ) y régimen preposicional (cuidar $+d e$ ), presentes en todas sus formas verbales y con todos los significados que pudieran desplegar.

Del total de casos obtenidos, se realizó una doble restricción. La primera, según criterios sintácticos, ya que se descartaron las construcciones cuyo objeto fuera un infinitivo ('ella cuida venir'), cláusulas encabezadas por 'que' ('ella cuida que vendrá'), participios ('lo tenía cuidado'), construcciones absolutas ('hay que cuidar') y otras preposiciones que no sean 'de + sustantivo' ('cuidar por el bien') o los casos en los que el 'de' sirve para introducir un constituyente que corresponde al elemento del cual se distancia el sujeto (del tipo 'cuidarse de las malas lenguas', que además es reflexivo) ${ }^{5}$. Respecto de las estructuras con 'se' ('cuidarse'), tanto antepuesto como pospuesto, cabe señalar que, debido a que responden al eje del análisis (que descansa sobre el rasgo de animacidad), estas fueron cotejadas con el fin de extraer información respecto de su funcionamiento sintáctico, pero finalmente descartadas para el análisis cuantitativo ${ }^{6}$.

Cálculo de $\chi^{2}: \mathrm{w}=0.1, \mathrm{~N}=1090.256, \mathrm{df}=3$, sig.level $=0.05$, power $=0.8$.

Puede llamar la atención que, según se aprecia en las Tablas 1 y 2, en ambos verbos las estructuras con CD y CPREP se documentan con escasa frecuencia en comparación con las demás. Sin embargo, cabe recordar que en este estudio se busca observar la estructura argumental, específicamente el análisis del segundo argumento. Y ni los infinitivos, cláusulas completas o participios corresponden a argumentos propiamente tal.

6 Si bien las estructuras con 'se' registran más apariciones que con CD o CPREP, no superan el 5\% del total en cada siglo, siendo, en su mayoría, casos problemáticos de interpretación. 
La segunda restricción se realizó según criterios filológicos, ya que se descartaron dos casos en que el texto en su contexto no era lo suficientemente claro, ya sea por la oscuridad de la sintaxis o porque el objeto directo podía ser comprendido en más de un sentido. Así, la muestra finalmente quedó conformada por 483 casos: 101 correspondientes al verbo cuidar ( 76 con CD y 25 con CPREP) y 382 al verbo curar (265 con CD y 117 con CPREP).

Se realizó posteriormente un muestreo por siglo y por estructura. De manera adicional, se buscaron las variantes grafológicas de ambos verbos, cuidar y curar, que pudieran aparecer en estos siglos como rasgos arcaizantes, a saber: cuydar, coitar y coidar en alternancia con cuidar; y curiar como variante de curar. De estas variantes, se registraron en el corpus solo cuydar y curiar con régimen directo y preposicional, de modo que el muestreo se realizó considerando las cuatro formas definitivas: cuydar, cuidar, curiar y curar. No obstante, para el análisis, se agruparon los casos de cuydar con cuidar, por un lado, y curar con curiar, por otro, dado que, en esencia, son el mismo verbo (i.e. mismo significado), pero con representaciones fonéticas y/o gráficas distintas según la época.

El análisis consistió en asignar a cada ejemplo los rasgos de diferenciación semánticos de definitud (/ \pm Definido/), especificidad (/₫Específico/), animacidad (/ \pm Animado/) y, en casos de objetos animados, humanidad (/ \pm Humano/). Así, una construcción con CD, como en (5), se caracteriza como /-Definido/, /-Específico/, /+Animado/, /-Humano/. Cabe señalar que el análisis se hizo en paralelo con la estructura del verbo curar para observar si existen diferencias que puedan ser explicadas por causas analógicas entre estos verbos de tan cercana naturaleza semántica.

(5) mas sabian de labrar canpos \& curar ganados (Ramírez de Guzmán, 1440-1460)

\section{Resultados}

A partir de los datos proporcionados por el corpus, previamente a la aplicación de las restricciones, las estructuras que se registran en periodo medieval para los verbos cuidar y curar son las siguientes: 
Tabla 1

Cantidad de estructuras que admite el verbo cuidar registradas en el CORDE

\begin{tabular}{lcccc}
\hline Cuidar & s. XII & s. XIII & s. XIV & s. XV \\
\hline Cuidar + inf. & $3(38 \%)$ & $223(15 \%)$ & $314(18 \%)$ & $612(13 \%)$ \\
Cuidar + 'que' & $1(13 \%)$ & $576(38 \%)$ & $918(52 \%)$ & $1.884(40 \%)$ \\
Participios & $3(38 \%)$ & $459(30 \%)$ & $325(18 \%)$ & $1.643(35 \%)$ \\
Cuidar + 'se' & 0 & $54(4 \%)$ & $85(5 \%)$ & $139(3 \%)$ \\
Absoluto & $1(13 \%)$ & $146(10 \%)$ & $111(6 \%)$ & $366(8 \%)$ \\
Cuidar + CD & 0 & $17(1 \%)$ & $20(1 \%)$ & $40(1 \%)$ \\
Cuidar + 'de' & 0 & $9(1 \%)$ & $6(0,3 \%)$ & $11(0,3 \%)$ \\
Otras prep. & 0 & $41(3 \%)$ & $45(3 \%)$ & $44(1 \%)$ \\
\hline Totales & $\mathbf{8}$ & $\mathbf{1 . 5 2 5}$ & $\mathbf{1 . 8 2 4}$ & $\mathbf{4 . 7 3 9}$ \\
\hline
\end{tabular}

Tabla 2

Cantidad de estructuras que admite el verbo curar registradas en el CORDE

\begin{tabular}{lccrr}
\hline Curar & s. XII & s. XIII & s. XIV & s. XV \\
\hline Curar + 'se' & $1(4 \%)$ & $2(3 \%)$ & $31(26 \%)$ & $276(52 \%)$ \\
Curar + CI & $3(14 \%)$ & $0(0 \%)$ & $0(0 \%)$ & $10(2 \%)$ \\
Curar + CD & $10(52 \%)$ & $58(84 \%)$ & $57(50 \%)$ & $140(27 \%)$ \\
Curar + 'de' & $0(0 \%)$ & $6(10 \%)$ & $22(18 \%)$ & $89(17 \%)$ \\
Otras const. & $7(30 \%)$ & $2(3 \%)$ & $7(6 \%)$ & $10(2 \%)$ \\
\hline Totales & 21 & 68 & 117 & 525 \\
\hline
\end{tabular}

1 Ya sean valores absolutos o preposiciones, incluyendo de con sentido de 'preocuparse por'.

\subsection{Régimen preposicional con de}

El verbo cuidar registra 25 ocurrencias en el corpus analizado con la estructura de régimen preposicional, mientras que curar registra 117. No se reconocen casos para cuidar ni curar sino hasta el siglo XIII. Sin embargo, a partir de entonces se comienzan a detectar curvas interesantes: curar exhibe una línea ascendente similar a la presentada con régimen directo, pero cuidar, no obstante, se mantiene en frecuencias bajas:

Tabla 3

Número de casos registrados por siglo con cuidar y curar con CPREP

\begin{tabular}{lccccc}
\hline CPREP & XII & XIII & XIV & XV & Total \\
\hline Cuidar & 0 & 9 & 6 & 10 & $\mathbf{2 5}$ \\
Curar & 0 & 6 & 22 & 89 & $\mathbf{1 1 7}$ \\
\hline
\end{tabular}


En cuanto a las propiedades semánticas encontradas con esta estructura, se presentan los rasgos /+Animado/, /+Humano/, /+Definido/ y /+Específico/, distribuidos de la siguiente manera7:

Tabla 4

Distribución de rasgos con CPREP a través de los siglos

\begin{tabular}{llccccc}
\hline & Rasgo & XII & XIII & XIV & XV & Total \\
\hline Cuidar & Animado & 0 & $5(56 \%)$ & $3(50 \%)$ & $6(60 \%)$ & $\mathbf{1 4}(\mathbf{5 6 \%})$ \\
& Humano & 0 & $5(56 \%)$ & $3(50 \%)$ & $6(60 \%)$ & $\mathbf{1 4}(\mathbf{5 6 \%})$ \\
& Definido & 0 & $3(33 \%)$ & $3(50 \%)$ & $1(10 \%)$ & $7(\mathbf{2 8 \%})$ \\
& Específico & 0 & $6(67 \%)$ & $3(50 \%)$ & $6(60 \%)$ & $\mathbf{1 5}(\mathbf{6 0 \%})$ \\
\multirow{6}{*}{ Curar } & Animado & 0 & $3(50 \%)$ & $13(59 \%)$ & $22(25 \%)$ & $\mathbf{3 8 ( 3 2 \% )}$ \\
& Humano & 0 & $2(33 \%)$ & $11(50 \%)$ & $19(21 \%)$ & $\mathbf{3 2 ( 2 7 \% )}$ \\
& Definido & 0 & $3(50 \%)$ & $2(9 \%)$ & $24(27 \%)$ & $\mathbf{2 9}(\mathbf{2 5 \%})$ \\
& Específico & 0 & $2(33 \%)$ & $12(55 \%)$ & $48(54 \%)$ & $\mathbf{6 2}(\mathbf{5 3 \%})$ \\
\hline
\end{tabular}

Referente al significado léxico que despliega la estructura, cabe mencionar que los 25 casos de cuidar con CPREP emplean el sentido medieval de 'pensar', tal como muestran los ejemplos (6a), (6b) y (6c); a su vez que los 117 casos de curar llevan el significado de 'cuidar', como se puede ver en (7a), (7b) y (7c):

(6a) amembrándome de Ti en mío lecho, en cada vela cuydando de Ti, car eres mi ayuda (Herman el Alemán, c. 1240-1272)

(6b) (si) simplemente la baptizó non cuydando de lo uno nin de lo ál, baptizado es (Cuéllar, 1325)

(6c) Amadís le dixo riendo: - Ellos cuidan de mí lo que no deven (Rodríguez, 1482-1492)

(7a) que tot pastor que curiare de .c. oueias a suso, de una cordera en el mes de mayo (Anónimo, 1227)

(7b) por esto los suyos no plegaron a la pelea, porque ell et su ermano no curauan de caualleros. (Fernández, 1376-1391)

(7c) los rreyes e grandes señores tanto curavan de sus cavallos como de sí mismos (Escavias, 1467-1475)

7 La distribución considera la cantidad de veces que se presentó el valor /+/ en cada siglo. Así, en el siglo XIII el rasgo /Animado/ se presenta 5 veces de los 9 casos registrados en dicho siglo, lo cual corresponde a un $56 \%$ de ocurrencia. Así, de los 25 casos totales que registra cuidar con CPREP en los siglos XII, XIII, XIV y XV, el 56\% fueron animados (14 casos). El mismo criterio se aplicó para el resto de los rasgos. 


\subsection{Régimen directo}

En comparación con la estructura con CPREP, el número de ejemplos con la construcción con $\mathrm{CD}$ es mayor, dibujando, de igual manera, una curva ascendente con el paso de los siglos. Por el lado de curar, se observa que registra su aparición más tempranamente que cuidar: a partir del siglo XII comienza a proyectar una curva ascendente, registrando para comienzos del Renacimiento (siglo XV en adelante) un vasto número de apariciones con esta estructura:

Tabla 5

Número de casos registrados por siglo con cuidar y curar con CD

\begin{tabular}{lccccc}
\hline CD & XII & XIII & XIV & XV & Total \\
\hline Cuidar & 0 & 16 & 20 & 40 & $\mathbf{7 6}$ \\
Curar & 10 & 58 & 57 & 140 & $\mathbf{2 6 5}$ \\
\hline
\end{tabular}

Respecto de las propiedades semánticas de la construcción con CD, la distribución de rasgos por siglo ocurre de la siguiente forma:

Tabla 6

Distribución de rasgos con CD a través de los siglos

\begin{tabular}{llccccc}
\hline & Rasgo & XII & XIII & XIV & XV & Total \\
\hline Cuidar & Animado & 0 & 0 & $1(5 \%)$ & $1(2.5 \%)$ & $\mathbf{2}(3 \%)$ \\
& Humano & 0 & 0 & 0 & $1(2.5 \%)$ & $\mathbf{1 ( 1 \% )}$ \\
& Definido & 0 & $9(52 \%)$ & $9(45 \%)$ & $28(70 \%)$ & $46(61 \%)$ \\
& Específico & 0 & $10(63 \%)$ & $16(80 \%)$ & $27(68 \%)$ & $\mathbf{5 3 ( 7 0 \% )}$ \\
Curar & Animado & $8(80 \%)$ & $29(50 \%)$ & $20(35 \%)$ & $58(41 \%)$ & $\mathbf{1 1 6 ( 4 4 \% )}$ \\
& Humano & $7(70 \%)$ & $12(21 \%)$ & $16(28 \%)$ & $57(41 \%)$ & $\mathbf{9 2 ( 3 5 \% )}$ \\
& Definido & $1(10 \%)$ & $34(59 \%)$ & $44(77 \%)$ & $89(64 \%)$ & $\mathbf{1 6 8 ( 6 3 \% )}$ \\
& Específico & $8(80 \%)$ & $24(41 \%)$ & $23(40 \%)$ & $51(37 \%)$ & $\mathbf{1 0 6 ( 4 0 \% )}$ \\
\hline
\end{tabular}

En cuanto al significado léxico que aparece con esta estructura, cabe destacar que 74 casos del verbo cuidar despliegan el significado de 'pensar', como puede verse en los ejemplos (8a), (8b) y (8c), mientras que 2 casos emplean el sentido actual de 'cuidar' (i.e. 'atender (a alguien o algo)'), tal como puede observarse en los ejemplos (9a) y (9b). Si bien se trata de un número bajo, estos 2 casos (presentes en el siglo XIV y XV respectivamente) son, en efecto, los únicos que presentan el rasgo /+Animado/. (8a) en diziendo la mentira da a entender que primero cuydo aquella mentira ante que la dixese. (Anónimo, 1293) 
(8b) dandolo a entender que del viene sinon uos que cuydastes esta razon. (Anónimo, 1345)

(8c) Si quisieres ser sabio, deves cuydar todas las cosas que deven acaescer e pensar en (Anónimo, 1430)

(9a) a ocho omes del abad, e a quatro omes que cuidan las bestias, que son por todos 33 omes (Anónimo, 1338)

(9b) fecho a sus caballeros, diziendo: - Por çierto yo cuido mis caballeros, que vos traigo a buena enpresa (García, 1471-1476)

Por su parte, el verbo curar aparece utilizado con el significado de 'cuidar' a lo largo de los siglos XII, XIII y XIV (ejemplos 10a, 10b y 10c). En el siglo XV, empero, el significado empleado en su mayoría corresponde al actual 'sanar' (11), presente en el 94\% de los casos en dicho siglo, y solo 9 casos $(6 \%)$ responden al sentido medieval empleado hasta entonces.

(10a) Tod pastor que ganado curiare, si el ganado perdiere, por quanto iurare el (Anónimo, 1179-1184)

(10b) de la Biblia que Josep cuando era de seze años curiava el ganado de su padre con sos hermanos (Alfonso X, 1275)

(10c) Otrosí, los andadores deuen curiar todos los presos los que el iúdez por calonia (Anónimo, 1300)

(11) E syn dubda muchas cosas ay que curan la epilensia. (Anónimo, 1495)

\subsection{Distribución en el tiempo de estructuras sintácticas y sig- nificado léxico}

Desde el punto de vista del comportamiento sintáctico de ambos verbos, la Tabla 7 muestra la distribución de ambas estructuras, cuya frecuencia es graficada para visualizar cómo cambia el empleo que hacen de una y otra construcción (Gráfico 1).

Tabla 7

Distribución de estructuras CD y CPREP 


\begin{tabular}{clccccc}
\hline \multicolumn{1}{c}{ Rasgo } & XII & XIII & XIV & XV & Total \\
\hline Cuidar & CD & $/$ & $(16) 64.0 \%$ & $(20) 76.9 \%$ & $(40) 80.0 \%$ & $(\mathbf{7 6}) \mathbf{7 5 . 2 \%}$ \\
& CPREP & $/$ & $(9) 36.0 \%$ & $(6) 23.0 \%$ & $(10) 20.0 \%$ & $(\mathbf{2 5}) \mathbf{2 4 . 7 \%}$ \\
& & $(0)$ & $(25)$ & $(26)$ & $(50)$ & $(\mathbf{1 0 1})$ \\
Curar & CD & $(10) 100 \%$ & $(58) 90.6 \%$ & $(57) 72.1 \%$ & $(140) 61.1 \%$ & $(\mathbf{2 6 5}) \mathbf{6 9 . 3 \%}$ \\
& CPREP & $/$ & $(6) 0.9 \%$ & $(22) 27.8 \%$ & $(89) 38.8 \%$ & $(\mathbf{1 1 7}) \mathbf{3 0 . 6 \%}$ \\
& & $(10)$ & $(64)$ & $(79)$ & $(229)$ & $(382)$ \\
\hline
\end{tabular}

\section{Gráfico 1}

Perfil de uso de las estructuras con CD y CPREP

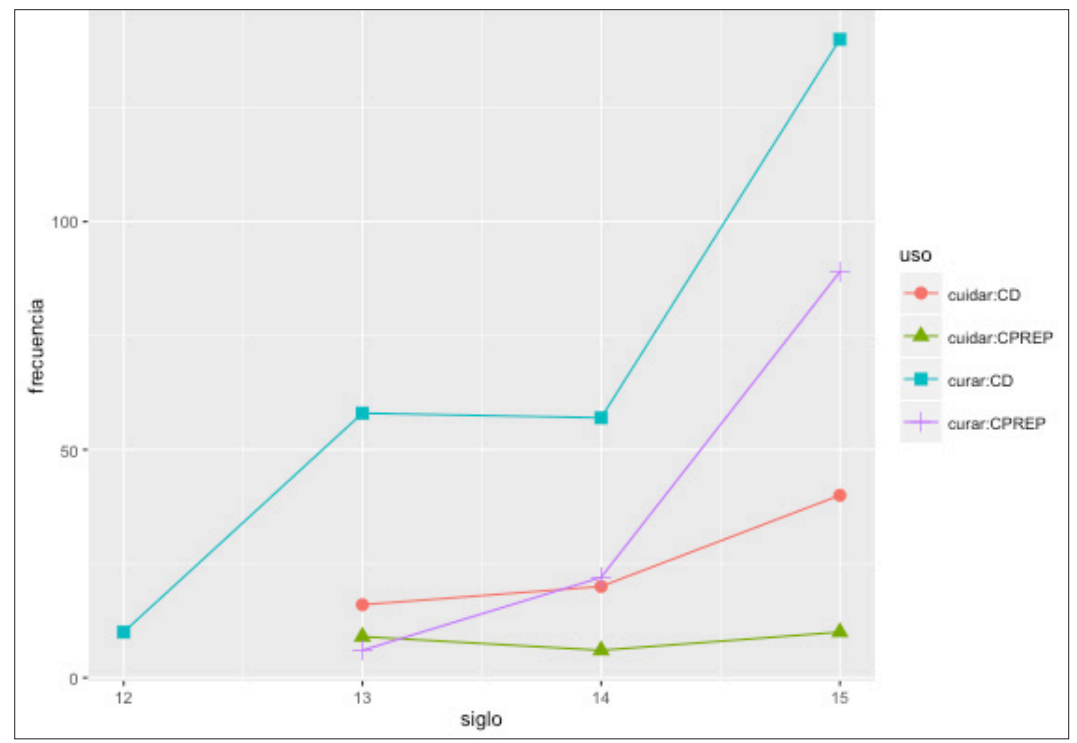


Respecto del significado léxico asociado a cada uno de los verbos, en el Gráfico 2, en concordancia con lo mencionado en la sección 4.1. y 4.2., se resume visualmente la tendencia a lo largo de los siglos respecto de la frecuencia de uso de los significados 'cuidar,' 'pensar' y 'sanar' asociados a los lexemas cuidar y curar. Como puede observarse, para finales de la Edad Media se alza el significado de curar 'sanar', mientras que emerge el de cuidar 'cuidar'.

\section{Gráfico 2}

Distribución del significado léxico en el tiempo

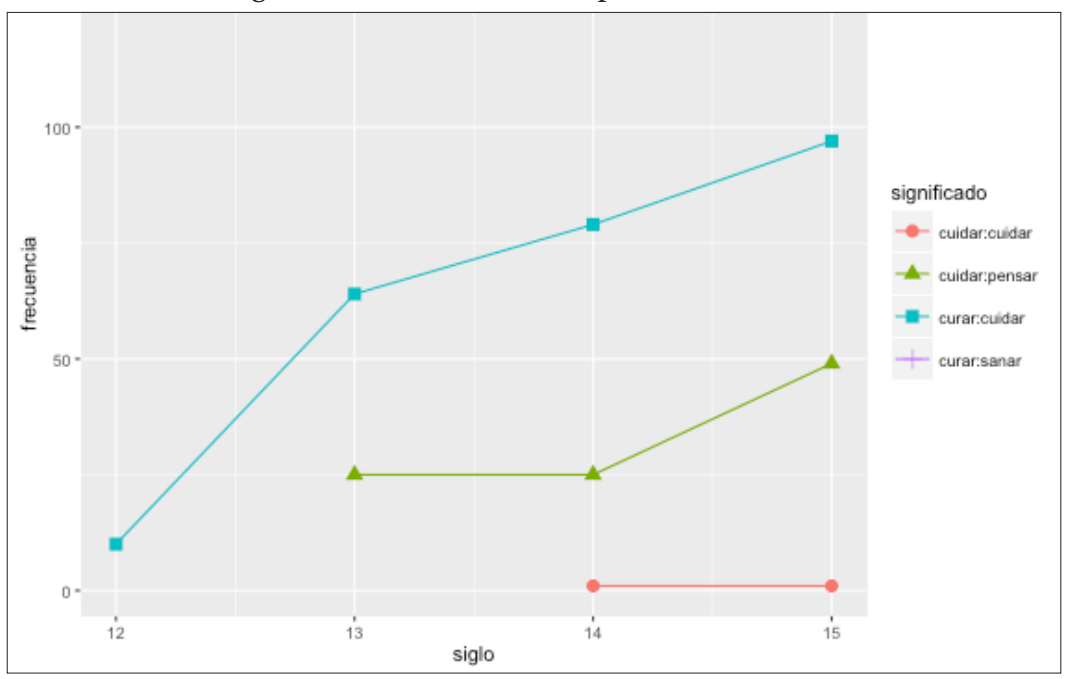

\section{Discusión de los datos}

Los resultados del análisis permiten hacer una serie de observaciones. En primer lugar, se puede afirmar que, si bien ambos verbos y estructuras se registran a lo largo de los siglos analizados, no lo hacen con la misma distribución (véase la Tabla 7). Estos resultados de distribución muestran, por un lado, que el patrón de frecuencia no es el mismo para todos los siglos y de manera significativa, puesto que $\chi^{2}(9)=36.5284, p=$ $.0015^{8}$; y, por otro, que a lo largo de la Edad Media ambos verbos favorecen

8 Si bien la línea ascendente que se incrementa con el tiempo está mediada, en parte, por el aumento de textos que el corpus ofrece para cada siglo (ciertamente se conservan menos textos en siglos antiguos que en épocas más modernas), la prueba estadística indica que la diferencia en el patrón de distribución supera lo esperado por el azar, de modo que dicha diferencia no solo está en este corpus analizado, sino que es generalizable. 
ampliamente al régimen directo. Sin embargo, cuidar tiene un aumento desde el siglo XIII (que parte con 64\%) hasta el siglo XV (que alcanza 80\%), mientras que curar disminuye drásticamente (del 100\% en siglo XII al 61.1\% en siglo XV). Y confirman, por otro lado, que el régimen preposicional asciende con curar (de $0.9 \%$ en el siglo XIII aumenta a $38.8 \%$ en el siglo XV, a costa del régimen directo), mientras que cuidar experimenta un ligero cambio en la dirección opuesta (de $36 \%$ en siglo XIII disminuye a $20 \%$ en siglo XV).

En segundo lugar, un análisis exhaustivo de los rasgos semánticos asociados al segundo argumento en cada una de las estructuras permite ver que existe una diferencia determinante entre cuidar y curar respecto del rasgo /+Animado/, ya que, si bien los dos verbos comparten los rasgos de humanidad, definitud y especificidad para la selección de sus argumentos objeto y en ambos tipos de estructuras, no ocurre lo mismo con la animacidad, ya que en uno de los verbos, curar, el segundo argumento presenta dicho rasgo con un valor positivo (/+Animado/) en ambas estructuras, mientras que en el otro no necesariamente (ver Tabla 6): cuidar reserva el rasgo /+Animado/ solo para el régimen preposicional, mientras que para el régimen directo presenta el rasgo /-Animado/ únicamente, salvo en dos ocasiones en que el verbo, además, proyecta otro significado léxico. Precisamente, los períodos en los que mayor concentración de casos de CD con el rasgo /+Animado/ hay con el verbo curar coinciden con los períodos en los que este verbo aún significa mayormente 'cuidar'.

Por estas dos razones (1. la ausencia del rasgo de animacidad en una estructura del verbo cuidar y 2. la presencia de este rasgo en asociación con un cambio de significado), se considera este rasgo el más significativo (en términos cualitativos) en comparación con los demás, de modo que se tomó como foco principal para el análisis y se prescindió de los otros.

El hecho de que no encontremos casos de animacidad con CD pero sí con CPREP en un mismo verbo (cuidar) permite hacer algunas observaciones importantes respecto de su posible grado de transitividad. Primero, algunos autores, Company (2014) por ejemplo, sostienen que una construcción preposicional en posición de segundo argumento implica una relación asimétrica entre dos miembros, propiciada por el sentido de 'origen o separación' de la preposición de. Esto otorgaría, según Rivas (2000), una nota de menor afección, lo que explicaría que el segundo argumento aparezca con el rasgo /-Animado/, en tanto el CPREP carecería de objeto efectuado (Rivas, 200o). Sin embargo, no parece clara 
la relación entre el distanciamiento del núcleo predicativo causado por la preposición y la animacidad del segundo argumento, puesto que la preferencia del rasgo de animacidad con CPREP demuestra que el segundo argumento es igualmente afectado por la acción verbal.

En este sentido, se podría hipotetizar que cuidar de es una construcción transitiva preposicional, esto es, "un complemento argumental de tema regido por preposición" (Candalija, 2013, p. 189) que afecta del mismo modo, en términos semánticos, al objeto como lo haría cualquier estructura transitiva tradicional con CD. Por tanto, no correspondería hablar de 'grados de afectación', sino del vestigio histórico de una preposición que relaciona el núcleo predicativo con su argumento adyacente.

Segundo, en cuanto al surgimiento del rasgo de animacidad del CD en dos casos tardíos con el verbo cuidar, es interesante ver que esto se produce precisamente en asociación con un cambio semántico, según el cual el verbo deja de ser estrictamente de actividad mental y adquiere el de 'atender (a alguien o algo).' Estos dos casos sugieren que de a poco empieza a producirse una transferencia semántica de curar hacia cuidar en los siglos XIV y XV, y que Corominas (1987) la ve establecerse recién en el siglo XVI.

Un dato interesante, si bien va más allá de los objetivos de la presente investigación, es la pérdida de la rección de CPREP por parte del verbo curar en español moderno, a pesar de que admitía tal estructura en español medieval. Esto demuestra que, más allá de la transferencia semántica experimentada por el verbo hasta el siglo XIV, luego se siguen produciendo cambios que evidentemente relacionan semántica y sintaxis. Probablemente, esto se deba a que el significado actual asociado a la forma (curar 'sanar') aparece en el siglo XV solo con la estructura con CD $y$, en cuanto comienza a preferir un cierto significado léxico, comienza a preferir también una cierta construcción.

\section{Conclusiones}

De nuestro análisis se puede concluir que, a diferencia de lo propuesto en trabajos previos, no es suficiente considerar el cambio semántico y la alternancia sintáctica de manera independiente, puesto que ambos fenómenos se relacionan estrechamente en el desarrollo de los verbos cuidar y curar. Así, la transferencia semántica de curar hacia cuidar posibilita e 
induce un cambio en el tipo de construcciones sintácticas admitidas, que va más allá de una simple pervivencia de estructuras, puesto que cuidar permite la admisión de objetos animados en construcciones de régimen directo solo cuando proyecta el significado actual 'cuidar'.

Este cambio ocurrido en el verbo cuidar es completamente compatible con aquel otro experimentado por curar a partir del siglo XV: a medida que cuidar fue asumiendo antiguos valores asociados originalmente a curar, este último pudo especializarse con el significado de 'sanar'. En efecto, "el paso de cuidar al sentido actual quizá se debiera a que la palabra que lo expresó durante mucho tiempo, curar, pasó a la esfera de la salud, dejando un hueco que ocupó el vocablo que nos interesa" (Santos y Espinosa, 1996, p.156). Se piensa que en algo pudo haber influido también el verbo pensar, que hasta fines de la Edad Media significaba 'cuidar', comportándose como sinónimo de cuidar (Cano, 1977, p.156).

Como se puede ver, todos estos cambios no se dan de manera aislada, sino dentro de un conjunto de verbos que comparten características, dentro del cual ciertos cambios pueden inducir otros. En este caso, al tratarse de verbos psicológicos o de actividad mental, sería ideal hacer un análisis general de todo el campo semántico a fin de ver con mayor claridad el panorama de cambios ocurridos.

Dicho esto, el análisis efectuado permite afirmar que, si bien no hay consenso entre los autores (cf. Cano, 1977; Rivas, 2000; García-Miguel, 1995) sobre la existencia de una diferencia, en términos semánticos, entre la construcción transitiva y la preposicional de cuidar en el castellano actual, es claro que sí la hubo, al menos, en época medieval, en tanto existe un rasgo semántico específico (/+Animado/) asociado al segundo argumento, que conlleva a un cambio en el comportamiento semánticosintáctico del verbo, condicionando, de esta manera, la aparición de cada una de las estructuras sintácticas: preposicional y no-preposicional. Así, cuidar empieza a seleccionar CD solamente con el rasgo /+Animado/ cuando recibe la transferencia semántica a partir de curar, lo cual sería posible gracias a que esta asociación estaba permitida en el verbo de origen. 


\section{Referencias bibliográficas}

ADESSE. Base de datos de Verbos, Alternancias de Diátesis y Esquemas Sintáctico-Semánticos del Español, [en línea]. Universidade de Vigo. < http://adesse.uvigo.es/data/verbos.php?sense=956>. [Consulta: 15 de mayo de 2016].

Alarcos, E. L. (1966). Verbo transitivo, verbo intransitivo y estructura del predicado. Archivum: Revista de la Facultad de Filología, (16), 5-17.

Baños, J. M. (2009). Sintaxis del latín clásico. Madrid: Liceus E-Excellence. Candalija, J. A. (2013). Transitividad preposicional: el doble complemento preposicional en español. Revista de Investigación Lingüística, 16, 177-207.

Cano, R. (1977). Cambios en la construcción de los verbos en castellano medieval. Archivum 27-28, 335-79.

. (1985). Sobre el régimen de las oraciones completivas en español clásico. Philologica hispaniensia: in honorem Manuel Alvar, Vol. 2, 81-94.

Company, C. (2014). “Las preposiciones de, des y desde”. Sintaxis histórica de la lengua española. Tercera parte: Adverbios, preposiciones y conjunciones. Relaciones interoracionales, (dir.), capítulo 12, Vol. 2.

CORDE. Banco de datos del Corpus diacrónico del español, [en línea]. <http://www.rae.es> [Consulta: 15 de mayo de 2016]

Corominas, J., y Pascual, J. A. (1987). Breve diccionario etimológico de la lengua castellana. Tercera edición muy revisada y mejorada. Madrid: Gredos.

CREA. Base de datos del Corpus de referencia del español actual, [en línea]. <http://www.rae.es> [Consulta: 15 de mayo de 2016].

Perseus Project. The Perseus Digital Library, [en línea]. Gregory Crane, Editor-in-Chief, Tufts University. <http://www.perseus.tufts. edu/hopper/>. [Consulta: 15 de mayo de 2016].

Dowty, D. (1991). Thematic proto-roles and argument selection. Language 67 (3), 547-619.

García-Miguel, J. M. (1995). Transitividad y complementación preposicional en español. Verba, anuario galego de filoloxía. Anexo 40. Universidade de Santiago de Compostela. 
García, J, M. (2006). Teoría y práctica: una distinción inquietante. Nómadas (14). Recuperado de: http://www.redalyc.org/articulo.oa?id=18101415

Glare, P. G. (1982). Oxford Latin dictionary (OLD). Clarendon Press. Oxford University Press.

Hopper, P. y Thompson, S. (1980). Transitivity in grammar and discourse. Language 56 (2). Pp. 251-299.

Luraghi, S., y Parodi, C. (2008). Key terms in syntax and syntactic theory. Bloomsbury Publishing.

Martínez, M. H. (1987). Sobre la rección y el régimen preposicional. Archivum: Revista de la Facultad de Filología.

Næss, Å. (2007). Prototypical transitivity. Vol. 72. John Benjamins Publishing Company.

Real Academia Española (2014). Cuidar. En Diccionario de la Lengua Española (23 a ed.). Recuperado de < http://dle.rae. es/?id=BbpgxqI>

Rivas, E. (2000). Régimen directo y régimen preposicional en castellano medieval. Philologica canariensia (6-7), pp. 117-144.

Santos, L. A. y Espinosa, R. M. (1996). Manual de semántica histórica. Madrid: Síntesis.

Torrego, M. E. (2010). Función textual y literaria de la pasiva en la Eneida de Virgilio. En Perfiles de Grecia y Roma. Actas del XII Congreso Español de Estudios Clásicos. Valencia, 22 al 26 de octubre de 2007 (pp. 195-227). 